\title{
V. EAR.
}

Otitis Externa Tropica-P. N. Gerrad-Lancet, September 23, I 899 .

The author gives a description of an affection which is of fairly common occurrence in Singapore and the Malay States. The symptoms somewhat resemble those associated with furtunculosis, although the discharge of pus from the outer ear may take place painlessly. The results of bacteriological examination of the discharge are given, and an antiseptic line of treatment is suggested.

\section{StClair Thomson.}

Deafness as a Result of the Abuse of Phenacetine-H. GuLEkl -Zcitschr. fiir Prak. Acrstc, November I 5, 1899.

The author gave a prescription for powders containing 0.7 of phenacetine, one powder to be taken twice daily. Through the stupidity of those in charge, the powders were given every two hours; so that in the course of less than 24 hours the patient took 7.0 of the drug.

Aside from other toxic symptoms, the patient was entirely deaf. On the subsidence of the general symptoms the deafness continued and has proved to be permanent. Meningitis could be excluded, and the only explanation is that the function of the ear was entirely destroyed by the phenacetine.

VITTUM.

The Politzer and Gruber Clinics-Johx P. Morson-The Canad. Pract. and Rer', Vol. xxiv, No. Io.

Dr. Morton, in a short article, details in a very interesting manner the methods of diagnosis employed in both of these noted clinics. Intwalia, he makes the following statements: "Electric headlights are not used for illumination of the ear." In the diagnosis of catarrhal adhesive processes in the middle ear, "Bing's ingenions test is used to test the mobility of the stapes. Through the medium of a Eustachian catheter, an ear trumpet is connected directly with the tympanic cavity; the sound waves are thus conducted immediately through the base of the stapes and membrana tympani secundaria to the labyrinthine fluids. Another trumpet is connected with the external meatus. If speech cannot be heard through the latter source, but can easily be heard when spoken into the tube connected with the catheter, the stapes is then judged to be freely movable and the malleus and incus to be in some way stiffened.

Dr. Bing has also instituted a method which aids in differentiating ear trouble from nose trouble. The sounding tuning fork is placed upon the mastoid process; as soon as the note ceases, the external meatus is closed with the finger, and if the sound is not again heard middle ear disease is diagnosed."

In auditory nerve disease, good perception for the high notes is considered of no value, for so many cases of pronounced nerve 
trouble are experienced in which excellent perception for the highest notes is retained. Rinne's test gives a positive result, the explanation being as follows: Normally the perception of the tuning fork through the air is longer than through the bone; in either case the nerve is finally called into action, and when it is diseased the air and bone conduction must suffer equally, which leaves them in the same relation to each other as in the normal condition, viz., positive Rinne. The important point, then, is not that we have a positive Rimne, for such is found in normal conditions, but that the Rinne is rendered positive by the shortening of the bone conduction."

Ginis Wishar'T.

\section{The Conservative Special Treatment of Chronic Suppuration of} the Middle Ear-Stetren-Berliner Klin. Wochenschr., September II-IS, I 899 .

This scholarly paper, which runs through two numbers of the journal mentioned above, deserves to be read by all who are interested in treating the ear, therefore, by all physicians. After remarking that not all cases of middle-ear trouble demand the radical operation, the author cites Lucac, who gives the results of his great experience: that, the general symptoms which justify the radical operation, are marked dizziness with disturbances in the gait, nausea and yomiting; while the local symptoms have to do with the cluration of the disease, the condition of the post-aural region, the frequency of attacks of pain, facial paralysis, and the nature and quantity of the discharge.

In a thoroughly moderate and scientific manner the atuthor then pleads for a more universal recourse to medical before proceeding to the heroic measures included in the radical operation. One great trouble met with in these cases was to find something which would render the thick tenacious pus more fluid so that the middle ear might be properly cleansed. The author thinks he has found this remedy in iodide of potash. His formula for the solution will be given later on. He also urges the use of Haug's chinolin-naphthol gauze, which on account of its softness and looseness of mesh can be gently packed deep into the meatus, and even into the middle ear itself, through the large perforation which is usually present in these cases. Granular conditions he combats with pure trichloracetic acid when the granulations are prominent. When merely a finely granular condition of the drumhead or middle ear exists he advises the insertion of a tampon saturated with a ro per cent solution of the acid.

To overcome the fetid and stinking condition of the discharge when present he makes use of menthoxol, a remedy which contains peroxide of hydrogen, and in contact with pus gives off oxygen, leaving behind menthol dissolved in alcohol. Most satisfactory results were obtained, the terrible fetor gradually disappearing, the discharge losing its deep yellow color and thin ichorous condition, and displaying the ordinary creamy color and consistence of laudable pus. The author himself sums up his treatment as follows: 